\title{
Multiobjective Bilevel Programming: concepts and perspectives of development
}

\author{
Maria João Alves ${ }^{1,3}$, Carlos Henggeler Antunes ${ }^{2,3}$, João Paulo Costa ${ }^{1,3}$ \\ ${ }^{1}$ CeBER and Faculty of Economics, University of Coimbra, Portugal \\ ${ }^{2}$ DEEC, University of Coimbra, Polo 2, Coimbra, Portugal \\ ${ }^{3}$ INESC Coimbra, Portugal \\ mjalves@fe.uc.pt; ch@deec.uc.pt; jpaulo@fe.uc.pt
}

\begin{abstract}
.
Bilevel programs model hierarchical non-cooperative decision processes with two decision makers, the leader and the follower, who control different sets of variables and have their own objective functions with interdependent constraints. Bilevel programs are very difficult to solve and even the linear case is NP-hard. In this chapter, a novel view on the main concepts in multiobjective and semivectorial bilevel problems is offered, including new types of solutions that are relevant for decision support. Optimistic and pessimistic leader's perspectives are explored; the extreme optimistic/deceiving and pessimistic/rewarding solutions in semivectorial problems and the optimistic Pareto fronts in multiobjective problems are defined and illustrated. Traditional and emerging application fields are reviewed. Potential difficulties and pitfalls associated with computing solutions to bilevel models with multiple objectives are outlined, shaping possible research avenues.
\end{abstract}

Keywords: multiobjective optimization; bilevel programming; semivectorial bilevel; optimistic vs. pessimistic approaches; optimistic, deceiving, pessimistic, rewarding solutions.

\section{Introduction}

Bilevel programs model hierarchical non-cooperative decision processes with two decision makers, the leader and the follower, who control different sets of variables and have their own objective functions with separate and/or interdependent constraints. The decision process is sequential: the leader first sets the values of his variables $x$ to optimize his objective function; the follower then reacts by choosing the values of his variables $y$ that optimize his objective function within the feasible solutions resulting from the leader's decision $x$. That is, the follower's problem is embedded in the leader's feasible region. Therefore, the leader needs to incorporate the follower's response into his optimization process because it affects solution feasibility and the leader's objective value. This type of sequential decision-making arises in many aspects of resource planning, management and policy-making, namely concerning the definition of pricing policies. For instance, in competitive electricity retail markets, retailers should establish commercial offers incorporating dynamic (time-of-use) tariffs aiming to maximize profits. However, they should take into account consumer's demand response to profit from time-differentiated tariffs by means of rescheduling the operation of appliances to minimize electricity bills (Alves and Antunes, 2018b).

The multiobjective bilevel problem (MOBP) may have multiple objective functions at one or both levels. A special case MOBP is the semivectorial bilevel problem (SVBP), in which there is a single objective function at the upper level and multiple objectives at the lower level. A set of lower level efficient solutions exists for each leader's decision $x$ whenever multiple objective functions are considered at the lower level. In these circumstances, the leader's decision process should anticipate the follower's reaction within his efficient solution set, i.e. foreseeing the follower's trade-off between the lower level multiple objectives for any instantiation of the $x$ variables. In the example mentioned above, to make the most of time-of-use tariffs, the consumer may want to minimize the electricity bill and also the discomfort associated with rescheduling the operation of appliances outside the habitual time slots. Some approaches assume that the leader knows the follower's utility function, i.e. the leader knows the follower's choice, thus enabling to reduce the problem to a single objective bilevel problem or to a bilevel problem with multiple objectives at the upper level only. These cases lead to identifying an optimal solution or an efficient solution set for the 
leader, respectively. However, this assumption is seldom realistic in practice, since the follower's reaction within his efficient solution set depends on a preference structure that is not known by the leader and may be difficult to elicit.

With no information about the efficient solution the follower will choose, the leader may adopt a more optimistic or a more pessimistic attitude in face of his expectation of the more or less favorable follower's choice. Therefore, different types of solutions resulting from distinct leader's attitudes and follower's reactions should be computed to offer information about possible outcomes and ranges of objective values.

Bilevel programs are very difficult to solve and even the linear case is NP-hard (Dempe, 2002). MOBP and SVBP become even more complicated due to the difficulties in computing solutions and issues associated with the decision process concerning the interaction leader - follower.

This chapter aims to offer a novel view on the main concepts in SVBP and MOBP, paying special attention to new types of solutions that are useful to support decisions based on this type of models. Optimistic and pessimistic leader's perspectives are explored, framing the extreme optimistic/deceiving and pessimistic/rewarding solutions in SVBP and the Optimistic Pareto front in MOBP, deriving from different leader's stances and follower's reactions, which require specific algorithmic techniques. Illustrative examples are used to shed light on these concepts, its relevance for decision support and the potential difficulties and pitfalls associated with computing solutions to bilevel models in a multiobjective setting.

In section 2, the single objective bilevel problem is formulated and the inducible region is defined. Then the MOBP is presented, paying attention to the particular case of SVBP. A brief literature review on algorithmic approaches is presented. Section 3 is devoted to bilevel problems with multiple objective functions at the upper level and a single objective at the lower level. Section 4 addresses bilevel problems with multiple objective functions at the lower level, defining the optimistic, deceiving, pessimistic and rewarding solutions for the SVBP and the Optimistic Pareto front in MOBP, offering illustrative examples of these concepts. Section 5 presents an overview of selected traditional and emerging application fields. Section 6 unveils pitfalls associated with the computation of solutions to the SVBP/MOBP and offers a view on possible research avenues, namely regarding the decision support framework. Concluding remarks are presented in Section 7.

\section{$2 \quad$ Multiobjective Bilevel Programming}

\subsection{Formulations and fundamental concepts}

A general bilevel programming problem with a single objective function at each decision level can be defined as follows:

$$
\begin{array}{ll}
\max _{x \in X} \quad & F(x, y) \\
\text { s.t. } & G(x, y) \leq 0 \\
& y \in \underset{y \in Y}{\arg \max _{y \in Y}}\{f(x, y): g(x, y) \leq 0\}
\end{array}
$$

$X \subseteq \mathbb{R}^{n_{1}}$ defines constraints only on the upper level decision variables $x$, which are controlled by the leader, and $Y \subseteq \mathbb{R}^{n_{2}}$ defines constraints only on the lower level decision variables $y$. $G(x, y) \leq 0$ and $g(x, y) \leq 0$ are general constraints, involving both $x$ and $y$, in the upper and the lower level problems. $F(x, y)$ and $f(x, y)$ represent the leader's and the follower's objective functions, respectively.

The follower's rational reaction set to a given $x^{\prime} \in X$ is:

$\Psi\left(x^{\prime}\right)=\left\{y^{\prime} \in \mathbb{R}^{n_{2}}: y^{\prime} \in \arg \max _{y \in Y}\left\{f\left(x^{\prime}, y\right): g\left(x^{\prime}, y\right) \leq 0\right\}\right\}$.

The set of feasible solutions over which the leader may optimize is called the inducible region (IR):

$$
I R=\left\{(x, y) \in \mathbb{R}^{n_{1}+n_{2}}: x \in X, G(x, y) \leq 0, y \in \Psi(x)\right\} .
$$

The bilevel problem is the problem seen by the leader. Quotation marks in "max" $F(x, y)$ express the unclear definition of the objective function value $F(x, y)$ from the leader's perspective (since he has control only over $x$ ) if the set of optimal solutions to the lower level problem is not singleton (Dempe, 2009). Even in the case where the lower level problem is a scalar optimization problem, more than one possible response of the follower may exist resulting from alternative optimal solutions to the follower's objective function. This poses a problem to the leader because the follower's choice may affect significantly the leader's 
decision. Most of the work on bilevel programming circumvents this difficulty by supposing that there is a single optimal solution to the lower level problem or adopting an optimistic approach. The optimistic approach presumes that the follower's response is always the most convenient for the leader. Under this assumption, the upper level optimization is executed with respect to $x$ and $y$, which means that the leader can influence the decision of the follower (Dempe, 2009). However, if the leader is risk-averse and wishes to limit the harm resulting from an undesirable option of the follower, a pessimistic approach should be considered. In this case the leader hedges against the worst case. Therefore, he chooses values for his variables that perform 'best' in view of the 'worst' follower's response (Tsoukalas, Wiesemann and Rustem, 2009). The pessimistic bilevel problem is even more difficult to solve than the optimistic one.

A general multi-objective bilevel programming problem with $k$ objective functions at the upper level and $m$ objective functions at the lower level can be formulated as follows:

$$
\begin{aligned}
& \max _{x \in X} \quad\left(F_{1}(x, y), \ldots, F_{k}(x, y)\right) \\
& \text { s.t. } \quad G(x, y) \leq 0 \\
& \quad y \in \arg \max _{y \in Y}\left\{\left(f_{1}(x, y), \ldots, f_{m}(x, y)\right): g(x, y) \leq 0\right\}
\end{aligned}
$$

The bilevel problems comprising only one objective function at the upper level $(k=1)$ and multiple objective functions at the lower level $(m \geq 2)$ has been called semivectorial bilevel problem. A general SVBP can be formulated as follows:

$$
\begin{array}{ll}
\text { "max" } & F(x, y) \\
\text { s.t. } & G(x, y) \leq 0 \\
& y \in \underset{y \in Y}{\arg \max _{y \in Y}}\left\{\left(f_{1}(x, y), \ldots, f_{m}(x, y)\right): g(x, y) \leq 0\right\}
\end{array}
$$

Only efficient (Pareto optimal) solutions to the lower level problem for each $x$-vector are feasible to the MOBP/SVBP. Let $Y(x)=\{y \in Y: g(x, y) \leq 0\}$. For a given $x^{\prime} \in X$, a solution $y^{\prime} \in Y\left(x^{\prime}\right)$ is efficient to the lower level problem (f-efficient) if and only if there is no other $y \in Y\left(x^{\prime}\right)$ that dominates $y^{\prime}$, i.e. such that $f_{j}\left(x^{\prime}, y\right) \geq f_{j}\left(x^{\prime}, y^{\prime}\right)$ for all $j=1, \ldots, m$, and $f_{j}\left(x^{\prime}, y\right)>f_{j}\left(x^{\prime}, y^{\prime}\right)$ for at least one $j$.

Therefore, the set of $f$-efficient solutions for a given $x^{\prime} \in X$ can be defined as: $\Psi_{\mathrm{Ef}}\left(x^{\prime}\right)=\left\{y^{\prime} \in Y\left(x^{\prime}\right)\right.$ : there is no $y \in Y\left(x^{\prime}\right)$ such that $\left.f\left(x^{\prime}, y\right) \succ f\left(x^{\prime}, y^{\prime}\right)\right\}$, where $\succ$ denotes the dominance relation.

$$
\begin{aligned}
& \text { The inducible region of the MOBP } \quad \text { or } \quad \text { the } \quad \text { SVBP } \\
& I R=\left\{(x, y) \in \mathbb{R}^{n_{1}} \times \mathbb{R}^{n_{2}}: x \in X, G(x, y) \leq 0, y \in \Psi_{\mathrm{Ef}}(x)\right\} \text {. }
\end{aligned}
$$

When multiple objectives are considered at the lower level, the follower has, in general, a set of efficient solutions for each leader's decision $x$. If the leader does not have information about the follower's choice (e.g., by knowing his utility function), the leader should prepare for any compromise solution selected by the follower within the lower level efficient solution set. This uncertainty about the follower's behavior requires that the leader should adopt an attitude in face of his expectation of the follower's choice, which may be more or less favorable according to the leader's single (SVBP) or multiple (MOBP) objectives. According to the more optimistic or more pessimistic attitude adopted by the leader anticipating the follower's choice, different types of solutions should be computed to offer information about possible outcomes and ranges of objective function values. In fact, it is not realistic to assume that the follower is indifferent to all efficient solutions obtained for a given decision of the leader or chooses according to the leader's objectives.

In a SVBP, the optimistic formulation considers that the solution to the lower level problem for each $x$ is the $f$-efficient solution leading to the best value of the upper level objective function; the pessimistic formulation considers that the solution to the lower level problem for each $x$ is the $f$-efficient solution that gives the worst value of the upper level objective function. In MOBP, the optimistic formulation has been almost exclusively used, which assumes that the solutions to the lower level problem for each $x$ are the $f$ efficient solutions leading to efficient solutions to the upper level problem. Only a few studies have addressed the uncertainty associated with the lower level decision (in the sense that, in principle, the leader does not have information about the follower's decision). The concept of pessimism is not clearly defined and further research is necessary on this topic. 
Four extreme solutions may be identified in the SVBP according to the more optimistic or more pessimistic leader's attitude: in the optimistic solution, the leader obtains the best objective function value when the follower decides in the best interest to the leader; however, the leader may engage in an optimistic attitude when setting his variables but the follower's choice is the worst for the leader, thus leading to the deceiving solution; in the pessimistic solution, the leader obtains the best objective function value when the follower's choice for each setting of upper level variables is the worst for the leader; however, the leader may engage in a pessimistic attitude when setting his variables but the follower's reaction is the most favorable to the leader, thus leading to the rewarding solution (Alves and Antunes, 2016).

\subsection{Classical and metaheuristic methods for bilevel with multiple objective functions}

The MOBP with multiple objective functions at the upper level and a single objective function at the lower level just requires the consideration of a set of efficient solutions to the upper level problem; therefore, the issues associated with the uncertainty of the follower's choice are not at stake (unless alternative optimal solutions to the lower level problem exist).

Calvete and Galé (Calvete and Galé, 2010b) addressed the bilevel linear problem with multiple objective functions at the upper level and a linear objective function at the lower level. When all objective functions are linear and constraints at both levels define polyhedra, the authors prove that the efficient solution set is non-empty and propose different scalarization techniques to obtain efficient points. These techniques involve solving linear bilevel problems with a single objective function at each level.

Alves et al. (Alves, Dempe and Júdice, 2012) proposed an exact procedure for bilevel linear programs with multiple objective functions at the upper level and a single objective function at the lower level, which is based on the reformulation of the problem as a multiobjective mixed 0-1 linear program that is dealt with a reference point algorithm. This procedure enables to characterize the whole Pareto front in bi-objective problems; in problems with any number of objective functions, a subset of nondominated solutions can be computed interactively according to the decision maker's preferences.

Alves and Costa (Alves and Costa, 2014) developed a multiple objective particle swarm optimization (PSO) algorithm to solve bilevel linear problems with multiple objective functions at the upper level, which aims to generate a good approximation of the whole Pareto front by using a hybrid strategy for the global best selection and an adaptive mutation operator.

Other approaches have been developed to deal with specific applications using bilevel models with multiple objectives at the upper level and a single objective at the lower level, e.g. the works of (Roghanian, Sadjadi and Aryanezhad, 2007) for supply chain planning and (Zhou et al., 2016) for a low-carbon power dispatch problem.

In SVBP, classical approaches and population-based metaheuristics have been developed mainly devoted to compute the optimistic solution. Classical approaches include using the Karush-Kuhn-Tucker (KKT) conditions, penalty methods and techniques exploring vertices in linear problems. The first approach involves replacing, whenever possible, a scalarization of the lower level problem by its KKT conditions. The second approach consists of formulating a nonlinear programming problem approximating the original one, which is solved iteratively by means of a penalty function method applied to the lower level problem leading, under certain conditions, to a sequence of approximated solutions converging to the optimal solution. The third approach is based on the property that only vertices of the constraint region need to be considered for the computation of the optimistic optimal solution to a linear SVBP. Population-based metaheuristics typically include evolutionary, PSO and differential evolution (DE) algorithms to cope with the difficulties to compute exact solutions.

The SVBP was first addressed by Bonnel (Bonnel, 2006), providing necessary optimality conditions for an optimistic formulation. Considering weakly efficient solutions to the lower level problem, Bonnel and Morgan (Bonnel and Morgan, 2006) proposed a solution approach based on a penalty function. Ankhili and Mansouri (Ankhili and Mansouri, 2009), Zheng and Wan (Zheng and Wan, 2011), Zheng et al. (Zheng, Chen and Cao, 2014), and Ren and Wang (Ren and Wang, 2016) developed penalty function methods to compute the optimistic solution to the SVBP with a multi-objective linear programming (MOLP) problem at the lower level. Calvete and Galé (Calvete and Galé, 2011) also focused on bilevel problems with a MOLP lower level problem (with all constraints linear and the upper level objective function quasiconcave). The problem is reformulated as an optimization problem over a nonconvex region given by the union of faces of the polyhedron defined by all constraints, so that an extreme point of the polyhedron is the optimistic solution to the problem. Both an enumerative exact algorithm and a genetic-based algorithm are proposed. Lv and Wan (Lv and Wan, 2014) proposed another algorithm for the linear SVBP using the weighted-sum scalarization to reformulate the linear SVBP into a special BP where the lower 
level is a parametric linear scalar problem. Then the BP is transformed into a single level nonlinear, nonconvex programming problem with a linear objective function.

Liu et al. (Liu et al., 2014) developed necessary optimality conditions for the pessimistic formulation of the SVBP, transforming the pessimistic SVBP into a generalized minimax optimization problem with constraints using a scalarization technique. Lv and Chen (Lv and Chen, 2016) proposed a discretization iterative algorithm to compute the pessimistic solution to a SVBP with a convex lower level problem without upper level variables in the constraints. Alves et al. (Alves, Antunes and Carrasqueira, 2015) firstly introduced the concept of deceiving solution and proposed an algorithm based on PSO to approximate the optimistic, pessimistic and deceiving solutions to the SVBP. Algorithms based on DE and PSO were proposed by (Alves and Antunes, 2018a) to compute those solutions, as well as the rewarding solution.

Concerning bilevel problems with multiple objectives at both levels, most studies have also considered the optimistic formulation.

Shi and Xia (Shi and Xia, 1997) proposed an interactive algorithm for the nonlinear MOBP, which simplifies the problem by transforming it into separate multi-objective decision-making problems at each level, using a satisfactoriness concept to model the leader's preferences. This work has been the basis for other models and algorithms with two or more levels that consider interactivity between the algorithm and the upper level decision maker to compute a satisfactory solution (Shi and Xia, 2001), (Abo-Sinna and Baky, 2007). Eichfelder (Eichfelder, 2010) presented new theoretical results for the nonlinear non-convex MOBP and proposed an algorithm to problems with two objective functions at both levels with one upper level variable. Pieume et al. (Pieume et al., 2011) introduced a surrogate single level MOLP problem aimed to generate the whole set of feasible solutions to the upper level problem for the optimistic formulation of the linear MOBP. Two approaches for obtaining efficient solutions are developed, depending whether the leader is able or not to express his preferences regarding his objective functions.

Deb and Sinha (Deb and Sinha, 2010) proposed a hybrid evolutionary algorithm with a local search phase, with self-adaptive population size based on Euclidean distance and termination criteria based on the hypervolume measure. Zhang et al. (Zhang et al., 2013) presented a hybrid PSO algorithm with crossover operator for high dimensional MOBP using an elitist strategy. The multiobjective PSO algorithm developed by Carrasqueira et al. (Carrasqueira, Alves and Antunes, 2015) pays special attention to the lower level optimization process for each upper level decision variable vector, so that solutions obtained are actually efficient; otherwise they are not feasible to the upper level problem. All these algorithms aim to approximate the whole Pareto front of the problem considering an optimistic formulation of the MOBP. Gupta and Ong (Gupta and Ong, 2015) proposed the transformation of the lower level problem into a single-objective problem using scalarization techniques with adaptive parameters, e.g. weights in a weighted-sum of the objective functions. The leader's problem is modified by incorporating the weights of the lower level objective functions into the upper level problem, which are handled in a similar manner as the original leader's decision variables. Therefore, the weights evolve through variation operators (e.g. mutation and crossover) in the upper level and enter into the lower level optimization as fixed parameters. This means that the leader is able to choose the weights of the follower's objectives that most benefits his interests, thus assuming an optimistic approach. Ruuska and Miettinen (Ruuska and Miettinen, 2012) proposed a procedure to construct new evolutionary algorithms for the optimistic MOBP by integrating an evolutionary multi-objective algorithm with a partial order that is compatible with bilevel optimization (Ruuska, Miettinen and Wiecek, 2012).

A different approach was adopted by Nishizaki and Sakawa (Nishizaki and Sakawa, 1999) in their interactive procedure, assuming that the leader has some subjective anticipation or belief of the follower's response. This anticipation may be optimistic, pessimistic or the leader knows the follower's preferences (e.g. arising from the past behavior of the follower).

More recently, Sinha et al. (Sinha et al., 2016) considered the decision uncertainty involved in modelling the follower's behavior and recognized that it is unrealistic to assume that the leader can decide the tradeoff the follower may choose. To handle this uncertainty, the authors assumed that the follower's preferences are characterized by a value function parameterized by an uncertain preference vector (e.g., a linear function with a stochastic weight vector for the different lower level objectives) and proposed a two-step approach: firstly, the leader uses his expectation about the follower's preferences to obtain the Pareto front for fixed parameters; then, the leader examines the extent of uncertainty by estimating a confidence region around the Pareto front previously obtained. Sinha et al. (Sinha, Malo and Deb, 2017) classify the MOBP into three different formulations. The first one is the optimistic formulation, which the authors call standard formulation. The second one considers deterministic decisions at lower level, assuming the leader has perfect information about the follower's preference structure. Using this preference information, which can be modelled as a value function, the lower level problem can be reduced to a single objective optimization 
problem. Whenever this information does not exist or cannot be elicited, the authors consider a third formulation in which the leader experiences lower level decision uncertainty, assuming that the follower's preferences are represented by a random variable (with a distribution that is parameterized by each upper level decision). Since this lower level uncertainty may have a significant impact on the upper level objective function(s), the leader should be aware of the consequences of distinct follower's decisions.

\section{Bilevel problems with multiple objective functions at the upper level and a single objective function at the lower level}

The consideration of multiple objective functions only at the upper level does not impose challenges as hard as the ones associated with the need to consider a set of efficient solutions to the lower level problem with respect to the uncertainty of the follower's choice. However, computational difficulties are at stake for the identification of Pareto optimal solutions. It should be noticed that even in the case of a linear bilevel problem (LBP) with multiple objective functions at the upper level and a single objective function at the lower level (MO-SO-LBP), the characterization of the upper level Pareto optimal solutions should take into account that (Alves, Dempe and Júdice, 2012):

- $\quad$ supported but also unsupported Pareto optimal solutions may exist;

- $\quad$ the set of Pareto optimal solutions may be not connected even if $I R$ is connected;

- the set of Pareto optimal solutions (or even weakly Pareto optimal solutions) may not be equal to the union of faces of the constraint region of the MO-SO-LBP, which includes all the constraints of the leader and the follower; that is, a face may be just partially Pareto optimal.

Example 1, in Fig. 1, illustrates a MO-SO-LBP with two objective functions at the upper level (Alves, Dempe and Júdice, 2012). $S$ denotes the constraint region.
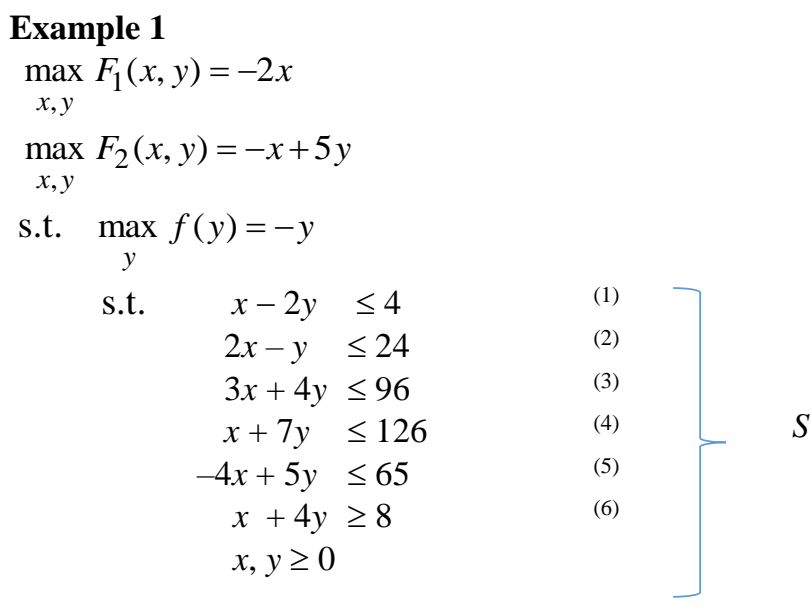


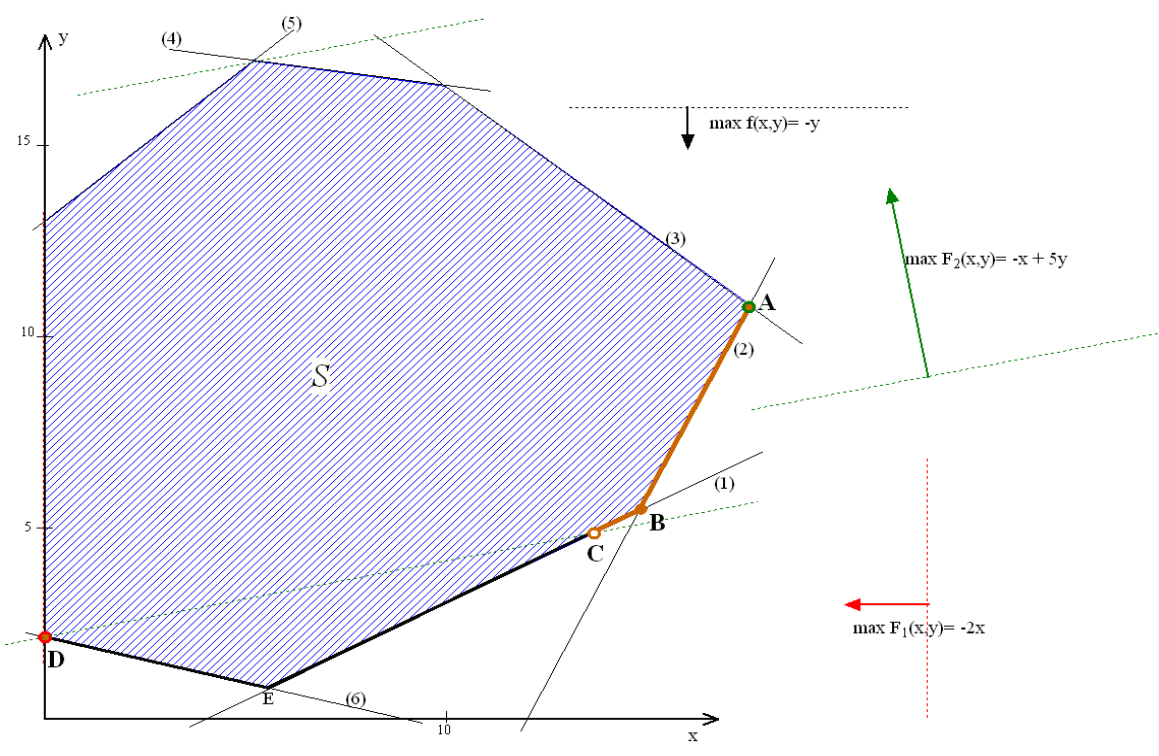

Figure 1. Graphical representation of Example 1.

The inducible region, $I R$, is $[\mathrm{DE}] \cup[\mathrm{EB}] \cup[\mathrm{BA}]$. The whole Pareto optimal solution set, which is a subset of $I R$, is $\{\mathrm{D}\} \cup] \mathrm{CB}] \cup[\mathrm{BA}]$. The values of the decision variables and the upper level objective functions in the points $\mathrm{A}, \mathrm{B}, \mathrm{C}$ and $\mathrm{D}$ are shown in Table 1.

Table 1. Values of the (weakly) Pareto optimal extreme points of Example 1

\begin{tabular}{l|cccc|} 
& $\mathrm{x}$ & $\mathrm{y}$ & $F_{1}$ & $\mathrm{~F}_{2}$ \\
\cline { 2 - 5 } $\mathrm{A}$ & 17.45455 & 10.90909 & -34.9091 & 37.09091 \\
$\mathrm{~B}$ & 14.66667 & 5.333333 & -29.3333 & 12 \\
$\mathrm{C}$ & 13.33333 & 4.666667 & -26.6667 & 10 \\
$\mathrm{D}$ & 0 & 2 & 0 & 10 \\
\hline
\end{tabular}

The analysis of Figure 1 enables to conclude that:

- $\quad$ D and A are the Pareto optimal solutions that maximize $F_{1}$ and $F_{2}$, respectively.

- Solutions from C (inclusive) to D (exclusive) of $I R$ are not Pareto optimal as they are dominated by $\mathrm{D}$. In comparison with $\mathrm{C}, \mathrm{D}$ is superior only in $F_{1}$ being equal in $F_{2}$. Hence, $\mathrm{C}$ is a weakly Pareto optimal solution. Solutions in $] \mathrm{DE}] \cup[\mathrm{EC}[$ are strongly dominated by $\mathrm{D}$.

- Only A and D are supported Pareto optimal solutions. All the others are unsupported, because there are convex combinations of $\mathrm{A}$ and $\mathrm{D}$ that would dominate them if they were feasible, i.e., if they belonged to $I R$.

This example shows that the Pareto optimal set of a MO-SO-LBP may be not connected and may have unsupported solutions. Furthermore, unsupported solutions may constitute the major part of the Pareto optimal set. Hence, they should not be disregarded.

\section{Bilevel problems with multiple objective functions at the lower level}

Let us first consider the bilevel problem with multiple objective functions at the lower level and one objective function at the upper level, i.e., the semivectorial bilevel problem SVBP.

\section{Example 2}

Consider the following problem with one objective function at the upper level and two objective functions at the lower level, whose constraint region $S$ is displayed in Figure 2. 
$\max F(x, y)=-x+5 y$

s.t.

$\max f_{1}(y)=-y$
$\max f_{2}(y)=x+y$

s.t.

$$
\begin{aligned}
& (1)-(6) \text { of Example } 1 \\
& x \leq 16
\end{aligned}
$$

Suppose the leader takes a particular decision, e.g. $x=5.3$. The follower's objectives become:

$\max f_{1}=-y ; \max f_{2}=5.3+y$. So, all the solutions in $\Psi_{\mathrm{Ef}}(5.3)$ represented in Figure 2 are efficient to the follower for this leader's decision.

For each leader's decision $x^{\prime}$ there is a set of efficient solutions $\Psi_{\mathrm{Ef}}\left(x^{\prime}\right)$ to the follower, which in this example are represented by vertical line segments delimited by the boundary of $S$. Therefore, for a given $x^{\prime}$ the following question arises: what will be the choice of the follower within $\Psi_{\mathrm{Ef}}\left(x^{\prime}\right)$ ?

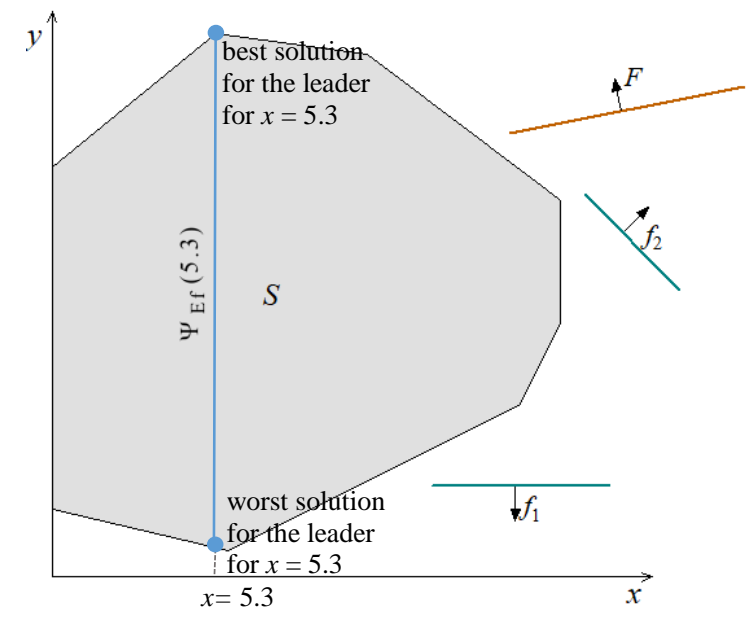

Figure 2 - Constraint region of Example 2

The two main ways suggested in the literature to frame the problem are the optimistic and the pessimistic approaches underlying different leader's perspectives. In the optimistic approach, the leader assumes that the follower is willing to support him and selects the solution among $\Psi_{\mathrm{Ef}}(x)$ that is the best for the leader.

- $\quad$ The optimistic solution, $\left(x^{\mathrm{o}}, y^{\mathrm{o}}\right)$, is given by

$$
\max _{x \in X, y \in Y}\left\{F(x, y): y \in \Psi_{\mathrm{Ef}}(x), G(x, y) \leq 0\right\} .
$$

In the pessimistic approach, the leader prepares for the worst case. The leader chooses the $x$ that leads to a solution with best $F$ in view of the follower's decisions $y$ worst for the leader.

- The pessimistic solution, $\left(x^{\mathrm{p}}, y^{\mathrm{p}}\right)$, if it exists, is given by

$$
\max _{x \in X}\left\{\min _{y \in Y}\left\{F(x, y): y \in \Psi_{\mathrm{Ef}}(x)\right\}: G(x, y) \leq 0\right\} .
$$

In addition to the optimistic and pessimistic solutions, other types of solutions can be defined that provide useful insights to the leader of possible outcomes and ranges of values resulting from different decisions. In particular, the following solutions may be relevant for decision support: the result of a failed optimistic approach - deceiving solution - and the result of a successful pessimistic approach - rewarding solution.

A deceiving solution results whenever the leader makes an optimistic decision and the follower's reaction is against the interests of the leader. Thus, given the optimistic upper level decision $x^{0}$,

- $\quad$ the deceiving solution is $\left(x^{\mathrm{d}}, y^{\mathrm{d}}\right)=\left(x^{\mathrm{o}}, y^{\mathrm{d}}\right)$ where $y^{\mathrm{d}}$ is given by $\min _{y \in Y}\left\{F\left(x^{\mathrm{o}}, y\right): y \in \Psi_{\mathrm{Ef}}\left(x^{\mathrm{o}}\right)\right\}$. 
Note that the deceiving solution may not satisfy upper level constraints $G(x, y) \leq 0$, i.e. it may be infeasible to the leader, because the aim is to show the follower's decision that is the worst outcome for the leader even if the follower does not take into account the upper level constraints. Nevertheless, this information can be useful to the leader's decision.

The rewarding solution is obtained whenever the leader takes a pessimistic approach and the follower's reaction is the most favorable to the leader. Thus, given the pessimistic upper level decision $x^{\mathrm{p}}$,

$$
\begin{aligned}
& \text { - the rewarding solution is } \quad\left(x^{\mathrm{r}}, y^{\mathrm{r}}\right)=\left(x^{\mathrm{p}}, y^{\mathrm{r}}\right) \quad \text { where } y^{\mathrm{r}} \text { is } \quad \text { given by } \\
& \max _{y \in Y}\left\{F\left(x^{\mathrm{p}}, y\right): y \in \Psi_{\mathrm{Ef}}\left(x^{\mathrm{p}}\right), G\left(x^{\mathrm{p}}, y\right) \leq 0\right\} \text {. }
\end{aligned}
$$

The rewarding solution is selected among the lower level efficient solutions for $x=x^{\mathrm{p}}$ that satisfy the upper level constraints. The aim is to show the follower's decision that is the best outcome for the leader, which should be feasible.

Let the follower's efficient solutions that are the best for the leader be called optimistic frontier and the follower's efficient solutions that are the worst for the leader be called pessimistic frontier. These frontiers are defined as follows.

Optimistic frontier: The optimistic frontier $(O f)$ is the set comprising the solutions $\left(x, y^{\prime}\right)$ such that $x \in X$ and $y^{\prime} \in O(x)=\left\{\arg \max _{y \in Y}\left\{F(x, y): y \in \Psi_{\mathrm{Ef}}(x), G(x, y) \leq 0\right\}\right\}$.

Pessimistic frontier: The pessimistic frontier $(P f)$ is the set comprising the solutions $\left(x, y^{\prime \prime}\right)$ such that $x \in X$ and $\mathrm{y}^{\prime \prime} \in P(x)=\left\{\underset{y \in Y}{\arg \min }\left\{F(x, y): y \in \Psi_{\mathrm{Ef}}(x)\right\}\right\}$.

The optimistic and the pessimistic solutions are the feasible solutions (i.e., which satisfy all constraints including $G(x, y) \leq 0$ ) with maximum value of $F$ in the optimistic frontier and the pessimistic frontier, respectively. The optimistic and rewarding solutions belong to $O f$, while the pessimistic and deceiving solutions belong to $P f$.

The value of $F$ in a feasible deceiving solution can be worse (and is never better) than the one in the pessimistic solution, because: the pessimistic solution $\left(x^{\mathrm{p}}, y^{\mathrm{p}} \in P\left(x^{\mathrm{p}}\right)\right)$ is the best feasible solution according to $F$ on the pessimistic frontier $P f$; the deceiving solution $\left(x^{\mathrm{d}}, y^{\mathrm{d}} \in P\left(x^{\mathrm{d}}\right)\right)$ also belongs to $P f$; thus, if the deceiving solution satisfies $G\left(x^{\mathrm{d}}, y^{\mathrm{d}}\right) \leq 0$, it cannot be better than the pessimistic solution, otherwise it would be the pessimistic solution itself. At the best, the deceiving solution is as good as the pessimistic solution.

Figure 3 shows the optimistic - rewarding and the pessimistic - deceiving solutions of Example 2 on the optimistic frontier $(O f)$ and the pessimistic frontier $(P f)$, respectively.

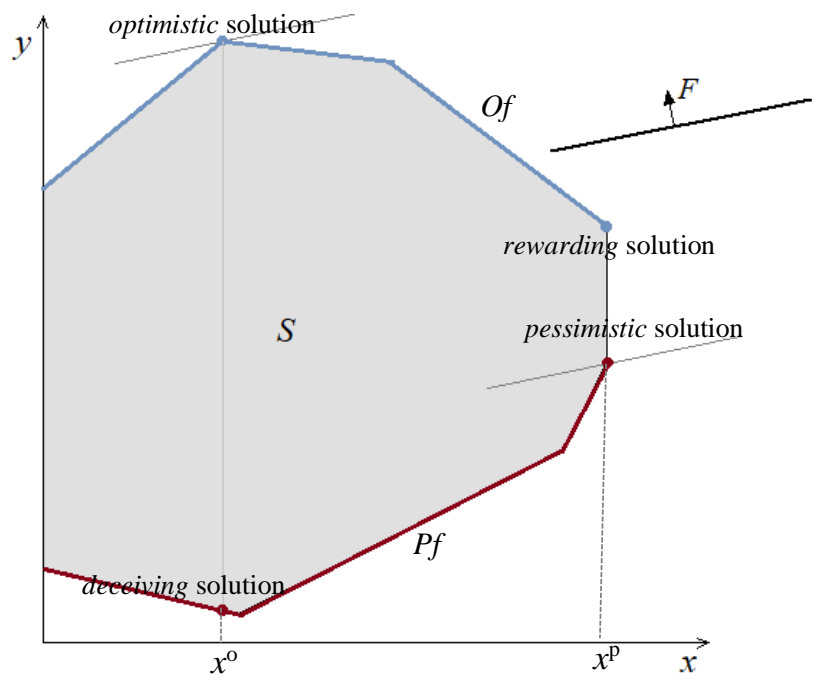

Figure 3 - Example 2: optimistic frontier $(O f)$ and pessimistic frontier $(P f)$; optimistic, pessimistic, deceiving and rewarding solutions. 
Let us consider another SVBL example with non-linear objective functions to be minimized. This problem is adapted from Problem 3 in (Deb and Sinha, 2009) considering just one upper level objective function, and it was presented in (Alves, Antunes and Carrasqueira, 2015).

\section{Example 3}

$$
\begin{array}{ll}
\min _{x} & F(x, y)=\left(y_{1}-1\right)^{2}+y_{2}^{2}+x^{2} \\
\text { s.t. } & \min _{y} f_{1}(x, y)=y_{1}^{2}+y_{2}^{2} \\
& \min _{y} f_{2}(x, y)=\left(y_{1}-x\right)^{2}+y_{2}^{2} \\
& \text { s.t. } \quad-1 \leq y_{1}, y_{2}, x \leq 2
\end{array}
$$

For a given value of $x$, the efficient solutions to the lower level problem are:

$$
\left(y_{1}, y_{2}\right) \in \mathbb{R}^{2}: \begin{cases}y_{1} \in[0, x], y_{2}=0 & \text { for } 0 \leq x \leq 2 \\ y_{1} \in[x, 0], y_{2}=0 & \text { for }-1 \leq x \leq 0\end{cases}
$$

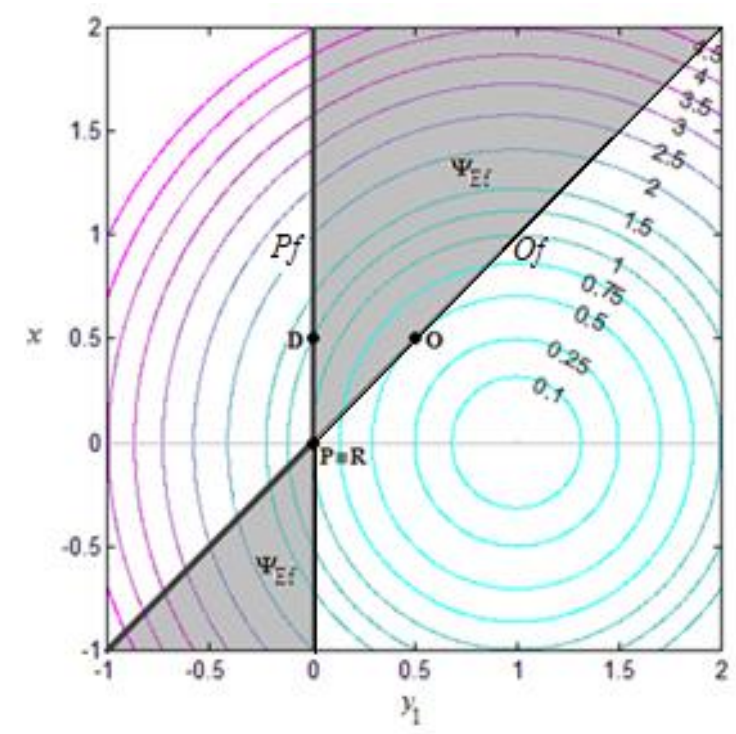

Figure 4 - Example 3: optimistic (O), pessimistic (P), deceiving (D) and rewarding (R) solutions.

In Fig. 4, $\Psi_{\mathrm{Ef}}$ denotes the union of $\Psi_{\mathrm{Ef}}(x)$ for all $x$, which is represented by the shaded area. The level curves of $F(x, y)$ are circles centered at the point $\left(y_{1}, x\right)=(1,0)$. Since $y_{2}=0$ for all efficient solutions to the lower level problem, only $y_{1}$ and $x$ are displayed in Fig. 4.

The optimistic solution is the point $\mathrm{O}:\left(x, y_{1}, y_{2}\right)=(0.5,0.5,0),\left(f_{1}, f_{2}\right)=(0.25,0)$ and $F=0.5$.

The solution that optimizes the leader's objective function within the subset of the follower's efficient solutions that are "worst for the leader", i.e. Pf, is the pessimistic solution (point P): $\left(x, y_{1}, y_{2}\right)=(0,0,0)$, $\left(f_{1}, f_{2}\right)=(0,0)$ and $F=1$.

The deceiving solution indicates the maximum risk the leader incurs if he adopts an optimistic approach, i.e. the leader chooses $x=0.5$ (point D): $\left(x, y_{1}, y_{2}\right)=(0.5,0,0),\left(f_{1}, f_{2}\right)=(0,0.25)$ and $F=1.25$.

If the leader takes a pessimistic approach (i.e. he chooses $x=0$ ), the only efficient solution to the follower is solution $\mathrm{P}$. Therefore, in this example, the leader cannot obtain a better value of $F$, which means that the rewarding solution $(\mathrm{R})$ coincides with the pessimistic solution.

In this problem, if the leader is willing to take some risk then the optimistic approach may be an interesting option because the worst outcome of the optimistic approach, given by the deceiving solution $(F=1.25)$, is not much worse than the pessimistic solution $(F=1.0)$; on the other hand, the optimistic solution provides a larger improvement to the leader's objective with respect to the pessimistic one $(F=0.5$ vs. 1.0). Moreover, there is no opportunity to obtain a solution better than the pessimistic one if the leader adopts a pessimistic approach, because there is only one efficient solution to the follower $(\mathrm{P} \equiv \mathrm{R})$. 
Concerning the bilevel problem with multiple objective functions at bot levels (MOBP), the aim of most procedures has been to approximate the whole upper level Optimistic Pareto front or they consider that the follower's preferences are known, thus reducing the MOBP to a BP with multiple objective functions at the upper level and a single objective function at the lower level.

A feasible solution $\left(x^{\prime}, y^{\prime}\right)$ to the MOBP is $F$-efficient if there is no other $(x, y) \in I R$ that dominates $\left(x^{\prime}, y^{\prime}\right)$, i.e. such that $F_{j}(x, y) \geq F_{j}\left(x^{\prime}, y^{\prime}\right)$ for all $j=1, \ldots, k$, and $F_{j}(x, y)>F_{j}\left(x^{\prime}, y^{\prime}\right)$ for at least one $j$. This definition assumes an optimistic approach in which the follower selects the $f$-efficient solutions that are efficient for the leader, thus leading to a subset of $I R$ containing all efficient solutions to the leader.

The Optimistic Pareto Front $(O P F)$ to the MOBP is composed of the $F$-efficient solutions $(x, y)$ whose $y$ are contained in the union of all $\Psi_{\mathrm{Ef}}(x)$ for every $x$. In bilevel problems with multiple objective functions at both levels, the concepts associated with the pessimistic approach are more difficult to define and may not be consensual.

Let us consider a MOBP example (Problem 3 in (Deb and Sinha, 2009)), which is the previous Example 3 with a second upper level objective function.

\section{Example 4}

$$
\begin{array}{cl}
\min _{x} & F_{1}(x, y)=\left(y_{1}-1\right)^{2}+y_{2}^{2}+x^{2} \\
\min _{x} & F_{2}(x, y)=\left(y_{1}-1\right)^{2}+y_{2}^{2}+(x-1)^{2} \\
\text { s.t. } & \min _{y} f_{1}(x, y)=y_{1}^{2}+y_{2}^{2} \\
& \min _{y} f_{2}(x, y)=\left(y_{1}-x\right)^{2}+y_{2}^{2} \\
& \text { s.t. } \quad-1 \leq y_{1}, y_{2}, x \leq 2
\end{array}
$$

The lower level efficient solutions are the same as in Example 3. The Optimistic Pareto Front $(O P F)$ is obtained for $y_{1}=x$ for $x \in[0.5,1]$ (Deb and Sinha, 2009).

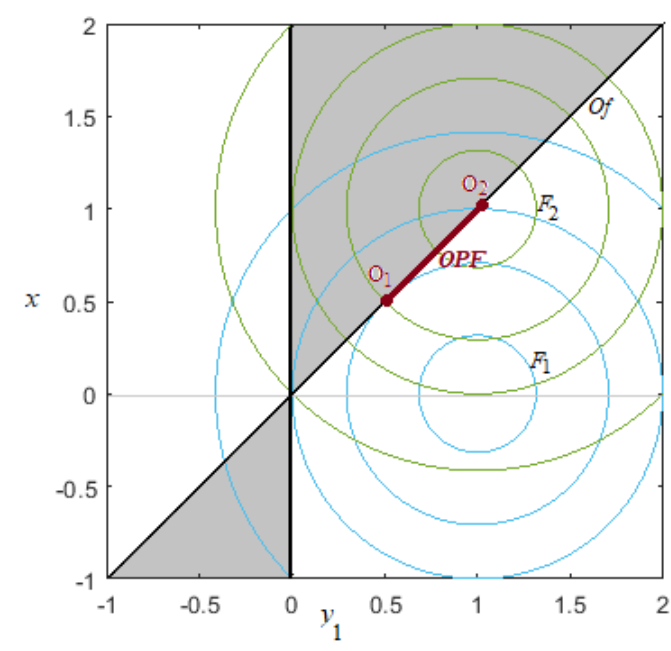

Figure 5 - Example 4: Optimistic Pareto Front $(O P F)$ in the variable space

In Fig. 5, the level curves of $F_{1}(x, y)$ and $F_{2}(x, y)$ are circles centered at the points $\left(y_{1}, x\right)=(1,0)$ and $\left(y_{1}\right.$, $x)=(1,1)$, respectively. If separately considered, the objective functions $F_{1}(x, y)$ and $F_{2}(x, y)$ have the same optimistic frontier $O f$, which is represented in Fig. 4. Therefore, the $O P F$ for the MOBP is located on the common Of between their individual optima $\left(\mathrm{O}_{1}\right.$ and $\left.\mathrm{O}_{2}\right)$.

Fig. 6 displays the upper level objective values for all solutions in Of, identifying the Optimistic Pareto Front $(O P F)$. 


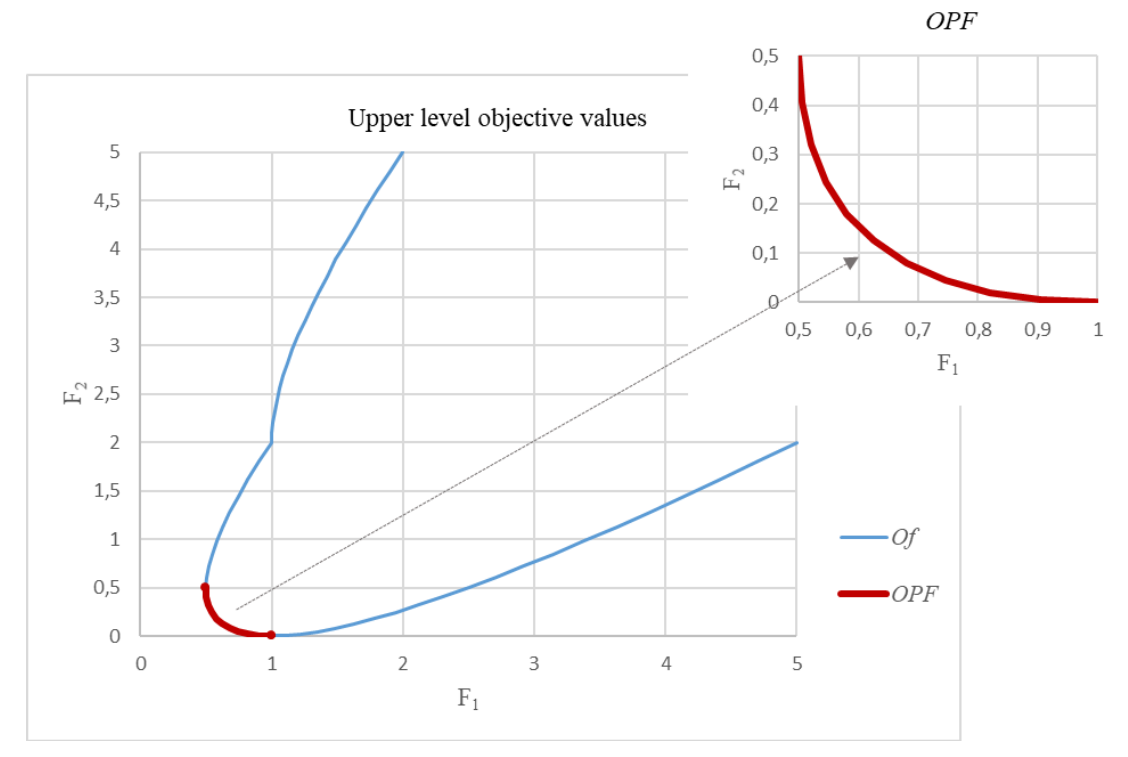

Figure 6 - Example 4: Optimistic Pareto Front $(O P F)$ in the upper level objective space

\section{$5 \quad$ Applications of MOBP}

Several configurations of multiobjective bilevel optimization models arise in the general framework of problems in which upper level decisions concern design and policy issues, while operational issues are at stake at the lower level. Areas of application include network design, transportation, facility location and logistics, security planning, energy systems, environmental management, process engineering, as well as structural, shape and/or layout design optimization. A brief review of representative traditional and emerging areas of applications is made below, with focus on the nature of the multiple objective functions at upper and/or lower levels.

\subsection{Network design, location and transportation policy}

Multi-objective bilevel models have been used in network design and transportation policy problems, namely concerning the optimization of tolls revenue, also considering congestion pricing in toll design, health and environmental issues. Sinha et al. (Sinha, Malo and Deb, 2015) developed a model for an authority managing a network of roads, whose objectives are maximizing toll revenues and minimizing pollution levels, while the network users aim to minimize travel cost and travel time. Chen et al. (Chen et al., 2010) presented a model for the network design problem in which the leader determines the optimal capacity enhancements in a transportation network by optimizing a set of system-wide objectives (traffic congestion, level of service and revenue) under demand uncertainty, while the lower level problem concerns the route choice behavior of users for a given capacity enhancement. Xu et al. (Xu, Tu and Zeng, 2012) dealt with a nonlinear minimum cost network flow problem in a large-scale construction project, in which in the upper level the construction contractor determines the material flow of each transportation network path aiming to minimize direct and transportation time costs and the lower level objective of the transportation manager is the minimization of transportation costs.

Gang et al. (Gang et al., 2015) proposed a model for the location of a stone industrial park with a hierarchical structure consisting of a local authority and stone companies. The local government is the leader aiming to minimize pollution emissions and development and operating costs. The stone companies are the followers aiming to minimize costs.

Hammad et al. (Hammad, Rey and Akbarnezhad, 2018) proposed a model to solve the multi-facility location problem with traffic equilibrium constraints. The aim is to locate a set of buildings with varying sensitivity thresholds due to the negative impacts of an existing semi-obnoxious facility. The traffic routing problem is modelled as a user equilibrium model at the lower level. 


\subsection{Environmental management}

Environmental economics and management decisions generally involve the interaction of a regulator authority/planning agency and individual firms maximizing profit but generating environmental externalities (e.g., air, land or water pollutants). Sinha et al. (Sinha et al., 2013) presented a study on environmental economics, in which the leader is the regulatory authority aiming to maximize total tax revenue and minimize the environmental damages caused by a mining company, which is the follower maximizing its total profit. Bostian at al. (Bostian, Whittaker, Sinha, et al. 2015a) considered the problem of designing a tax to reduce fertilizer use in agricultural production, in which the policy maker aims to minimize total fertilizer use in an agricultural watershed area and maximize total profits from agricultural production. For each tax rate, individual producers choose the quantity of fertilizer that maximizes their profit. Bostian et al. (Bostian, Whittaker, Barnhart, et al., 2015) evaluated the tradeoff between agricultural production and water quality considering the maximization of total profit and minimization of basin-level nitrate runoff resulting from fertilizer usage as the upper level objectives, while producer profit maximization is the lower level objective. The same objective functions at both levels are used in the model proposed by Whittaker et al. (Whittaker et al., 2017), also in a context of a single leader (the government authority establishing a policy) and multiple followers (the farmers who should comply with the policy in a way that maximizes their own objectives).

\subsection{Process engineering}

Process engineering deals with the design, operation, control and optimization of industrial processes, generally continuous ones, namely within chemical, petrochemical, agriculture, mineral processing, pharmaceutical, food and biotechnological industries. The use of multi-objective bilevel models depends on the type of processes and physical variables involved. In general, upper levels objectives are related with design and the lower level objectives with operation. Linnala et al. (Linnala et al., 2012) considered the design of a paper mill and its operation. At the design (upper) level, the objective functions to minimize are the fill percentages of wet broke and dry broke towers (in a broke system the rejected paper is collected and re-circulated back into the process as a raw material). At the operational (lower) level, the objective functions are the minimization of production loss, variation of fill percentage of the dry broke tower and variation of fill percentage of the wet broke tower, and the maximization of broke dosage. Halter and Mostaghim (Halter and Mostaghim, 2006) presented a thermodynamic model to determine parameters in the quantification of the physical and chemical properties of silicate melts. The upper level consists of minimizing the free energy of solid and liquid and minimizing the difference between the temperature obtained in the chemical reaction and the absolute temperature. In the lower level, the aim is the speciation data for each parameter set in the upper level.

\subsection{Security planning}

Li et al. (Li, Xu, et al., 2015) proposed a model for the construction site security problem in which the upper level problem involves maximizing the efficiency of the construction facilities system and minimizing the countermeasure cost and economic loss due to the facilities to be secured. In the lower level problem, the attacker aims to inflict maximum loss of efficiency in the construction facilities system. Li et al. (Li, Shen, et al., 2015) developed a model for the dynamic construction site layout and security planning problem. The objective functions in upper level problem are the minimization of the layout costs and consequences of a potential attack. In the lower level problem, the attacker aims to maximize the economic consequence of the attack to the construction facilities system.

\subsection{Energy systems}

A diversified set of applications in the energy sector has been reported in the literature, including tariff setting, electricity markets, and distributed generation. Zhou et al. (Zhou et al., 2016) proposed a model for a power dispatch problem in which the upper level decision maker is the regional power grid corporation, which allocates power quotas, and the lower level decision makers are power generation groups. The leader aims to maximize profit, minimize surplus power by balancing supply and demand and minimize total carbon emissions. Each follower establishes his power generation plans and prices to maximize profit. Lv et al. (Lv, Ai and Zhao, 2016) developed a model for the operation of a distribution network with gridconnected micro-grids. The upper (distribution network) level determines the optimal dispatch to optimize 
power losses and voltage profile. The lower (micro-grid) level uses the dispatch requirements to minimize the operation cost of distributed generators. Gao et al. (Gao et al., 2017) presented a coordinated planning model for the interaction of distributed generation and distribution network frames. The objective functions at the upper level are the minimization of the cost of integrated investment and operation maintenance, active power losses, power purchasing and power failure. The objective functions at the lower level are the minimization of the cost of distributed generation investment and operation maintenance, the expected deviation rate of voltage and the maximization of loss reduction and power generation of distributed generation.

Wang et al. (Wang, Wang and Cui, 2014) dealt with power consumption of data centers, proposing energy and locality aware multi-job scheduling in cloud computing to improve the energy efficiency of servers. The upper level objective functions are the minimization of the differences between each server's resource utilization and its optimal value and the maximization of data locality ratio. The lower level objective function is the minimization of the sum of the differences between each server's CPU utilization after scheduling and its optimal utilization. Stojiljković (Stojiljković, 2017) presented a case study of the design of a trigeneration system for an urban system. The upper level design objectives are the minimization of total costs, primary energy and greenhouse gases emission. The lower level operational objective deals with cost minimization.

Hawthorne and Panchal (Hawthorne and Panchal, 2014) dealt with feed-in-tariff policy design for decentralized energy infrastructure. The upper level policy designer aims to maximize the quantity of energy generated and minimize policy cost. The lower level stakeholders decide on quantities to maximize net present value and minimize capital investment seeking a Nash equilibrium resulting from market interactions. Alves and Antunes (Alves and Antunes, 2018b) developed a model for the interaction between electricity retailers and consumers to optimize electricity time-of-use retail pricing. The retailer (upper level decision maker) establishes dynamic time-of-use electricity prices to maximize profits. The consumer (lower level decision maker) responds by determining an appliance operation schedule to minimize the electricity bill and the dissatisfaction in face of his preferences and requirements.

\subsection{Logistics -production-distribution planning}

Jia et al. (Jia, Wang and Fan, 2014) address production-distribution planning problems. The leader is the distribution company aiming to minimize its overall cost. The follower is the manufacturer aiming to minimize the cost and the storage cost.

Calvete and Galé (Calvete and Galé, 2010a) considered the problem of a distribution company owning distribution centers, which acquires products from manufacturing plants and delivers them to retailers. The distribution company, at the upper level, aims to minimize the overall transportation costs. The manufacturing plants, at the lower level, decide the allocation of production to plants aiming to minimize production costs and the cost of underutilization of plant capacity.

\subsection{Structural design optimization}

In structural design optimization, the optimal design problem is solved at the upper level, in general minimizing the cost or weight of a structure, while the structural analysis problem is solved at the lower level, which may involve optimizing forces, energy, displacements, etc. Ghotbi (Ghotbi, 2016) dealt with the design of a high-speed mechanism considering kinematic and dynamic objectives, which are treated as the leader in different problem variants. The kinematic objectives include the minimization of the structural error and the deviation of the transmission angle from its ideal value. The dynamic objective consists of the minimization of the peak torque required to drive the input link over a cycle. Dandurand et al. (Dandurand et al., 2014) addressed the design of a hybrid vehicle layout at vehicle and battery levels. At the upper level, the layout of components in the under-hood of the vehicle is optimized, while the design of the battery pack is optimized at the lower level considering thermal and location criteria.

\section{$6 \quad$ Perspectives in multiobjective bilevel programming}

Multiobjective bilevel programming is currently a subject of important research efforts from different communities, ranging from mathematical programming to (population-based) metaheuristic approaches. It has also received increasing attention from different application areas as there are many practical situations involving hierarchical optimization problems with multiple objective functions at one or both levels. 
However, several conceptual and computational challenges remain, particularly with respect to the general properties of MOBP/SVBP and their impact on the development of effective algorithms.

Improving classical algorithms (using branch \& bound, enumeration or penalty techniques or KKT conditions), for certain classes of bilevel programming problems (e.g. linear or with lower-level convex problems), has the potential to trigger advances in multiobjective bilevel problems. Concerning approximation algorithms (heuristics or meta-heuristics) when dealing with practical applications, the consideration of the intrinsic characteristics of the application can lead to more efficient algorithms and improve the accuracy of the final solutions. In addition, the combination of optimization techniques with well-tried techniques from other fields, such as machine learning, is also a promising research avenue.

Still, one of the main challenges in dealing with MOBP/SVBP concerns the feasibility of solutions. When approximation algorithms are used on multiobjective optimization problems (with a single level), there is no guarantee that true efficient solutions are obtained, but the feasibility of the solutions can be easily assessed by checking the constraints. In MOBP/SVBP, solutions that are not efficient to the lower level problem do not belong to the inducible region, i.e. they are not feasible to the bilevel problem. The possibility of getting solutions whose feasibility status is not easily recognized makes the problem more difficult to handle.

Regarding applications, several multiobjective formulations that have been proposed in the literature may be reformulated with advantage as bilevel models due to their intrinsic hierarchical relationship.

This section details some pitfalls associated with the evaluation of solutions and algorithm performance in SVBP/MOBP, a view on research avenues and novel applications with focus in the area of smart grids.

\subsection{Pitfalls}

Due to the theoretical and computational difficulties to solve the SVBP and MOBP, meta-heuristic approaches have gained particular relevance, namely the ones based on the evolution of a population of solutions (genetic/evolutionary algorithms, particle swarm optimization, differential evolution). However, the inherently approximate nature of these techniques may lead to misleading results. In fact, since only efficient solutions to the lower level problem are feasible to the bilevel problem, approaches based on metaheuristics may yield apparently better solutions to the SVBP/MOBP but these solutions are invalid because they are not truly efficient to the lower level problem, although they may constitute good approximations. This may lead the algorithm to choose these solutions over truly efficient solutions, because they are better to the leader, presenting at the end of the computation process solutions that seem of good quality but are unfeasible. Indeed, this is an important pitfall that researchers must be aware of when comparing algorithms for SVBP/MOBP, both for the computation of the optimistic solution/optimistic Pareto front and for other types of solutions: a better front will not mean better performance of the algorithm if the efficiency of lower level solutions is not ensured. Although this drawback is intrinsic to these problems, whenever possible hybrid approaches may avoid it by coordinating meta-heuristics with good computational performance for the upper level search with exact mathematical programming algorithms to solve the lower level problem for each instantiation of the upper level variables.

Moreover, even if only efficient solutions to the lower level problem are obtained, the assessment of pessimistic and deceiving solutions in SVBP is not straightforward since those solutions may not belong to the pessimistic frontier (i.e., they are not the worst for the leader for the corresponding setting of upper level decision variables) and therefore are not valid.

Similar difficulties arise in the computation of a pessimistic upper level Pareto front in MOBP. In this setting, one possibility is considering that the follower's decisions that are included in the pessimistic upper level Pareto front are the most dominated for the leader. For instance, consider two follower's efficient solutions for the same $x:\left(x, y^{1}\right)$ and $\left(x, y^{2}\right)$ with $F\left(x, y^{1}\right) \succ F\left(x, y^{2}\right)$; then, a pessimistic approach would assume that the follower chooses $y^{2}$, the worst option for the leader. However, the identification of these most dominated solutions may be computationally difficult.

\subsection{Dealing with the leader's risk vs. opportunity}

As mentioned in Example 1, in SVBP the leader may take a high risk engaging in an optimistic attitude if the deceiving solution is significantly worse than the pessimistic one. In addition, if he engages in a pessimistic attitude, he still has the opportunity to obtain the rewarding solution, which may be close to the optimistic one, as in Example 1. Therefore, it is important for decision support purposes to offer the leader other types of solutions in addition to the four extreme ones proposed, that is, moderate solutions providing the highest expected value considering an optimism/pessimism index (e.g., probabilities of the follower's 
decision being in favor or against the interests of the leader). Another approach would be considering different plausible settings of the follower's preferences. The computation and comparison of solutions to SVBP/MOBP for these preferences would provide relevant information regarding balancing the risk and the opportunity associated with each leader's decision in face of the follower's possible choices.

\subsection{Difficulties of the pessimistic approach}

Almost all algorithms developed thus far for SVBP/MOBP have focused on the computation of the optimistic solution/Pareto front. As far as we know, there is no algorithm to compute the pessimistic Pareto front in MOBP. However, as mentioned above, considering only optimistic solutions may be unrealistic in many practical situations because this approach presumes that the follower does not have preferences and is indifferent to all efficient solutions obtained for a given decision of the leader.

Therefore, an emerging research field is the development of effective algorithms capable of computing not only the optimistic solution/optimistic Pareto front, but also the pessimistic solution/pessimistic Pareto front and other solutions that can offer the leader information about the risk vs. opportunity provided by adopting a given strategy.

\subsection{Novel applications in smart grids}

SVBP and MOBP are very relevant for decision support in actual decision problems, in which design and policy decision are at stake in the upper level and operational decisions should then be made at the lower level, possibly involving different stakeholders with potential conflicting interests.

An emerging application area in the realm of smart grids, in which consumer empowerment becomes a crucial issue, is the definition of dynamic tariffs as a component of the portfolio offers of a retailer (the leader) considering variable wholesale prices and network status. Consumers (the followers) confronted with time-differentiated prices are expected to engage in demand response actions by rescheduling appliance operation to less expensive periods and/or resetting thermostats thus trading-off cost and comfort dimensions. New applications of SVBP/MOBP optimization entail, for instance, managing congestion at distribution transformers by harnessing the flexibility associated with the charge/discharge of electric vehicle batteries as well as enabling load aggregator entities to participate in ancillary services or capacity markets. In these problems, the consideration of multiple followers, which may share decision variables, objective functions and constraints, is of utmost importance, since in the leader's outcome may be affected by the relationships among followers.

\section{$7 \quad$ Concluding remarks}

In this chapter we presented the main concepts of multiobjective bilevel problems, in which multiple objective functions may arise in each level problem or in both. When multiple objective functions are at stake in the lower level problem, uncertainty regarding the follower's choice within his efficient solution set should be considered. Optimistic and pessimistic leader's perspectives were explored by characterizing the extreme optimistic/deceiving and pessimistic/rewarding solutions in semivectorial bilevel problems and the Optimistic Pareto front in multiobjective bilevel problems, which result from different leader stances and follower reactions. Their relevance for decision support as well as the potential difficulties and pitfalls associated with computing solutions to bilevel models in a multiobjective setting were discussed. Traditional and emerging application areas were reviewed and perspectives of development in multiobjective bilevel optimization were outlined.

Acknowledgment. This work was supported by projects UID/MULTI/00308/2013, ESGRIDS (POCI-010145-FEDER-016434) and MAnAGER (POCI-01-0145-FEDER-028040).

\section{$8 \quad$ References}

Abo-Sinna, M. A. and Baky, I. A. (2007) 'Interactive Balance Space Approach for Solving Multi-level Multi-objective Programming Problems', Information Sciences, 177(16), pp. 3397-3410. doi: 
10.1016/j.ins.2007.02.005.

Alves, M. J. and Antunes, C. H. (2016) 'An illustration of different concepts of solutions in semivectorial bilevel programming’, in 2016 IEEE Symposium Series on Computational Intelligence (SSCI), pp. 1-7. doi: 10.1109/SSCI.2016.7850219.

Alves, M. J. and Antunes, C. H. (2018a) A differential evolution algorithm to semivectorial bilevel problems, Machine Learning, Optimization, and Big Data. MOD 2017. Lecture Notes in Computer Science. Edited by G. Nicosia et al. Springer, Cham. doi: 10.1007/978-3-319-72926-8_15.

Alves, M. J. and Antunes, C. H. (2018b) 'A semivectorial bilevel programming approach to optimize electricity dynamic time-of-use retail pricing', Computers and Operations Research, 92. doi: 10.1016/j.cor.2017.12.014

Alves, M. J., Antunes, C. H. and Carrasqueira, P. (2015) 'A PSO Approach to Semivectorial Bilevel Programming: Pessimistic, Optimistic and Deceiving Solutions', in Proceedings of the Genetic and Evolutionary Computation Conference (GECCO 2015), pp. 599-606. doi: 10.1145/2739480.2754644.

Alves, M. J. and Costa, J. P. (2014) 'An algorithm based on particle swarm optimization for multiobjective bilevel linear problems', Applied Mathematics and Computation. Elsevier, 247, pp. 547561.

Alves, M. J., Dempe, S. and Júdice, J. J. (2012) 'Computing the Pareto frontier of a bi-objective bi-level linear problem using a multiobjective mixed-integer programming algorithm', Optimization. Taylor \& Francis, 61(3), pp. 335-358.

Ankhili, Z. and Mansouri, A. (2009) 'An exact penalty on bilevel programs with linear vector optimization lower level', European Journal of Operational Research, 197(1), pp. 36-41. doi: 10.1016/j.ejor.2008.06.026.

Bonnel, H. (2006) 'Optimality conditions for the semivectorial bilevel optimization problem', Pacific Journal of Optimization, 2(3), pp. 447-468.

Bonnel, H. and Morgan, J. (2006) 'Semivectorial bilevel optimization problem: penalty approach', Journal of Optimization Theory and Applications, 131(3), pp. 365-382. doi: 10.1007/s10957-006-9150-4.

Bostian, M., Whittaker, G., Sinha, A., et al. (2015) 'Incorporating data envelopment analysis solution methods into bilevel multi-objective optimization', in 2015 IEEE Congress on Evolutionary Computation (CEC), pp. 1667-1674.

Bostian, M., Whittaker, G., Barnhart, B., et al. (2015) 'Valuing water quality tradeoffs at different spatial scales: An integrated approach using bilevel optimization', Water Resources and Economics. Elsevier, 11, pp. 1-12.

Calvete, H. and Galé, C. (2011) 'On linear bilevel problems with multiple objectives at the lower level', Omega, 39(1), pp. 33-40. doi: 10.1016/j.omega.2010.02.002.

Calvete, H. I. and Galé, C. (2010a) 'A multiobjective bilevel program for production-distribution planning in a supply chain', in Multiple Criteria Decision Making for Sustainable Energy and Transportation Systems. Springer, pp. 155-165.

Calvete, H. I. and Galé, C. (2010b) 'Linear bilevel programs with multiple objectives at the upper level', Journal of Computational and Applied Mathematics, 234(4), pp. 950-959. doi: https://doi.org/10.1016/j.cam.2008.12.010.

Carrasqueira, P., Alves, M. J. and Antunes, C. H. (2015) 'A Bi-level Multiobjective PSO Algorithm', in Gaspar-Cunha, A., Antunes, C. H., and Coello Coello, C. (eds) Evolutionary Multi-Criterion Optimization (EMO 2015), Lecture Notes in Computer Science 9018. Springer International Publishing, pp. 263-276.

Chen, A. et al. (2010) 'Stochastic multi-objective models for network design problem', Expert Systems with Applications, 37(2), pp. 1608-1619. doi: https://doi.org/10.1016/j.eswa.2009.06.048.

Dandurand, B. et al. (2014) 'Bilevel multiobjective packaging optimization for automotive design', Structural and Multidisciplinary Optimization. Springer, 50(4), pp. 663-682.

Deb, K. and Sinha, A. (2009) 'Solving bilevel multi-objective optimization problems using evolutionary algorithms', in Proc. of EMO 2009, LNCS 5467. Springer, pp. 110-124.

Deb, K. and Sinha, A. (2010) 'An efficient and accurate solution methodology for bilevel multi-objective programming problems using a hybrid evolutionary-local-search algorithm', Evolutionary computation.

Dempe, S. (2002) 'Foundations of bilevel programming'.

Dempe, S. (2009) 'Bilevel programming: implicit function approach', in Floudas, C. A. and Pardalos, P. M. (eds) Encyclopedia of Optimization. Boston, MA: Springer US, pp. 260-266. doi: 10.1007/978-0387-74759-0_44.

Eichfelder, G. (2010) 'Multiobjective bilevel optimization', Mathematical Programming.

Gang, J. et al. (2015) 'A multi-objective bi-level location planning problem for stone industrial parks', Computers \& Operations Research. Elsevier, 56, pp. 8-21. 
Gao, Y. et al. (2017) 'Multi-Objective Bilevel Coordinated Planning of Distributed Generation and Distribution Network Frame Based on Multiscenario Technique Considering Timing Characteristics', IEEE Transactions on Sustainable Energy. IEEE, 8(4), pp. 1415-1429.

Ghotbi, E. (2016) 'Multi-objective optimization of mechanism design using a bi-level game theoretic formulation', Concurrent Engineering. SAGE Publications Sage UK: London, England, 24(3), pp. 266274.

Gupta, A. and Ong, Y. (2015) 'An evolutionary algorithm with adaptive scalarization for multiobjective bilevel programs', in 2015 IEEE Congress on Evolutionary Computation (CEC), Sendai, 2015, pp. 16361642.

Halter, W. and Mostaghim, S. (2006) 'Bilevel optimization of multi-component chemical systems using particle swarm optimization', in 2006 IEEE Congress on Evolutionary Computation (CEC), pp. 12401247.

Hammad, A. W. A., Rey, D. and Akbarnezhad, A. (2018) 'A Bi-level Mixed Integer Programming Model to Solve the Multi-Servicing Facility Location Problem, Minimising Negative Impacts Due to an Existing Semi-Obnoxious Facility', in Data and Decision Sciences in Action. Springer, pp. 381-395.

Hawthorne, B. and Panchal, J. H. (2014) 'Bilevel formulation of a policy design problem considering multiple objectives and incomplete preferences', Engineering Optimization. Taylor \& Francis, 46(7), pp. 964-986.

Jia, L., Wang, Y. and Fan, L. (2014) 'Multiobjective bilevel optimization for production-distribution planning problems using hybrid genetic algorithm', Integrated Computer-Aided Engineering. IOS Press, 21(1), pp. 77-90.

Li, Z., Shen, W., et al. (2015) 'Bilevel and multi-objective dynamic construction site layout and security planning', Automation in Construction. Elsevier, 57, pp. 1-16.

$\mathrm{Li}, \mathrm{Z}$., Xu, J., et al. (2015) 'Bilevel multi-objective construction site security planning with twofold random phenomenon', Journal of Industrial \& Management Optimization, 11(2), pp. 595-617.

Linnala, M. et al. (2012) 'Bi-level optimization for a dynamic multiobjective problem', Engineering Optimization. Taylor \& Francis, 44(2), pp. 195-207.

Liu, B. et al. (2014) 'Optimality conditions for pessimistic semivectorial bilevel programming problems', Journal of Inequalities and Applications, 2014, p. 41. doi: 10.1186/1029-242X-2014-41.

Lv, T., Ai, Q. and Zhao, Y. (2016) 'A bi-level multi-objective optimal operation of grid-connected microgrids’, Electric Power Systems Research. Elsevier, 131, pp. 60-70.

Lv, Y. and Chen, J. (2016) 'A Discretization Iteration Approach for Solving a Class of Semivectorial Bilevel Programming Problem', Journal of Nonlinear Sciences \& Applications (JNSA), 9(5), pp. 28882899.

Lv, Y. and Wan, Z. (2014) 'A solution method for the optimistic linear semivectorial bilevel optimization problem', Journal of Inequalities and Applications, 2014(1), p. 164. doi: 10.1186/1029-242X-2014-164.

Nishizaki, I. and Sakawa, M. (1999) 'Stackelberg solutions to multiobjective two-level linear programming problems', Journal of Optimization Theory and Applications, 103(1), pp. 161-182.

Pieume, C. et al. (2011) 'Solving Bilevel Linear Multiobjective Programming Problems', American Journal of Operations Research, 1, pp. 214-219. doi: 10.4236/ajor.2011.14024.

Ren, A. and Wang, Y. (2016) 'A novel penalty function method for semivectorial bilevel programming problem', Applied Mathematical Modelling, 40(1), pp. 135-149. doi: 10.1016/j.apm.2015.04.041.

Roghanian, E., Sadjadi, S. J. and Aryanezhad, M. B. (2007) 'A probabilistic bi-level linear multiobjective programming problem to supply chain planning', Applied Mathematics and Computation, 188(1), pp. 786-800. doi: https://doi.org/10.1016/j.amc.2006.10.032.

Ruuska, S. and Miettinen, K. (2012) 'Constructing evolutionary algorithms for bilevel multiobjective optimization', in 2012 IEEE Congress on Evolutionary Computation (CEC), pp. 1-7.

Ruuska, S., Miettinen, K. and Wiecek, M. M. (2012) 'Connections between single-level and bilevel multiobjective optimization', Journal of Optimization Theory and Applications, 153(1), pp. 60-74.

Shi, X. and Xia, H. (1997) 'Interactive bilevel multi-objective decision making', Journal of the operational research society, 48(9), pp. 943-949.

Shi, X. and Xia, H. (2001) 'Model and interactive algorithm of bi-level multi-objective decision-making with multiple interconnected decision makers', Journal of Multi-Criteria Decision Analysis, 10, pp. $27-34$.

Sinha, A. et al. (2013) 'Multi-objective stackelberg game between a regulating authority and a mining company: A case study in environmental economics', in 2013 IEEE Congress on Evolutionary Computation (CEC), pp. 478-485.

Sinha, A. et al. (2016) 'Solving Bilevel Multicriterion Optimization Problems With Lower Level Decision Uncertainty', IEEE Transactions on Evolutionary Computation, 20(2), pp. 199-217. doi: 
10.1109/TEVC.2015.2443057.

Sinha, A., Malo, P. and Deb, K. (2015) 'Transportation policy formulation as a multi-objective bilevel optimization problem', in 2015 IEEE Congress on Evolutionary Computation (CEC), pp. 1651-1658. doi: 10.1109/CEC.2015.7257085.

Sinha, A., Malo, P. and Deb, K. (2017) 'Evolutionary Bilevel Optimization: An Introduction and Recent Advances', in Bechikh, S., Datta, R., and Gupta, A. (eds) Recent Advances in Evolutionary Multi-objective Optimization. Springer, Cham, pp. 71-103.

Stojiljković, M. M. (2017) 'Bi-level multi-objective fuzzy design optimization of energy supply systems aided by problem-specific heuristics', Energy. Elsevier, 137, pp. 1231-1251.

Tsoukalas, A., Wiesemann, W. and Rustem, B. (2009) 'Global optimisation of pessimistic bi-level problems', Fields Institute Communications, 55, pp. 1-29.

Wang, X., Wang, Y. and Cui, Y. (2014) 'A new multi-objective bi-level programming model for energy and locality aware multi-job scheduling in cloud computing', Future Generation Computer Systems. Elsevier, 36, pp. 91-101.

Whittaker, G. et al. (2017) 'Spatial targeting of agri-environmental policy using bilevel evolutionary optimization', Omega. Elsevier, 66, pp. 15-27.

$\mathrm{Xu}, \mathrm{J} ., \mathrm{Tu}, \mathrm{Y}$. and Zeng, Z. (2012) 'A nonlinear multiobjective bilevel model for minimum cost network flow problem in a large-scale construction project', Mathematical Problems in Engineering. Hindawi, 2012(Article ID 463976), p. 40 pages. doi: 10.1155/2012/463976.

Zhang, T. et al. (2013) 'Solving high dimensional bilevel multiobjective programming problem using a hybrid particle swarm optimization algorithm with crossover operator', Knowledge-Based Systems, 53, pp. $13-19$.

Zheng, Y., Chen, J. and Cao, X. (2014) 'A Global Solution Method for Semivectorial Bilevel Programming Problem', Filomat, 28(8), pp. 1619-1627.

Zheng, Y. and Wan, Z. (2011) 'A solution method for semivectorial bilevel programming problem via penalty method', Journal of Applied Mathematics and Computing, 37(1-2), pp. 207-219. doi: 10.1007/s12190-010-0430-7.

Zhou, X. et al. (2016) 'Low-carbon based multi-objective bi-level power dispatching under uncertainty', Sustainability. Multidisciplinary Digital Publishing Institute, 8(6), p. 533. 